\title{
Spontan, gewaltsam, von Hunger getrieben? \\ Diskursive Rahmung von food riots und Protesten gegen hohe Preise \\ in Burkina Faso 2008
}

\author{
Bettina Engels
}

Im Zuge der Nahrungsmittelpreiskrise 2008 fanden in vielen Städten weltweit und insbesondere in Afrika Hungeraufstände statt. Der Beitrag argumentiert, dass solche Aufstände häufig als spontan und gewaltsam dargestellt werden - nicht nur von ,westlichen' Medien und staatlichen Akteuren, sondern auch von oppositionellen Gruppen in den jeweiligen Ländern selbst. Der Artikel analysiert den Fall Burkina Fasos, wo Gewerkschaften und Bewegungsorganisationen im Anschluss an die Hungeraufstände zu umfangreichen Protesten gegen hohe Nahrungsmittelpreise mobilisierten. Diese Proteste und die Hungeraufstände unterschieden sich hinsichtlich der beteiligten sozialen Gruppen und der eingesetzten Protestrepertoires. Die etablierten oppositionellen Gruppen nutzten die riots als diskursiven Bezugspunkt, um ihre Strategien und Forderungen zu legitimieren und voranzubringen

Ersteinreichung: 23. Juni 2015; Veröffentlichung online: 1. Mai 2016

An english abstract can be found at the end of the document.

Inwiefern sind riots als kollektives politisches Handeln zu verstehen, als spezifische Form des Protests marginalisierter sozialer Akteure - oder als potenziell kriminelle Akte, spontan, ohne politische Ziele? Diese Frage wurde in den letzten Jahren intensiv etwa anlässlich der riots in Großbritannien vom August 2011, in den französischen Banlieues im Oktober und November 2005, in Stockholm 2013 und an vielen anderen Orten diskutiert. Die Bezeichnung als ,unpolitisch' oder ,kriminell' delegitimiert riots und legitimiert ein breites Spektrum repressiver und vermeintlich präventiver Maßnahmen. Riots als inhärent politisches Phänomen zu fassen betont hingegen ihre strukturellen Ursachen: ökonomische, politische und gesellschaftliche Prozesse wie Marktliberalisierung, die Kommodifizierung sozialer Grundversorgung, Einschnitte im Bereich des Wohlfahrtsstaats und diskriminierende Praktiken von Verwaltung und Polizei. Bis vor Kurzem wurde die Frage, inwiefern riots als politisches Phänomen zu fassen seien, fast ausschließlich zwischen diesen beiden Polen diskutiert. In jüngster Zeit richtet sich das Interesse verstärkt auch auf den politischen Subjektstatus von an riots aktiv Beteiligten (Trott 2013).

Im Mittelpunkt dieses Beitrags stehen institutionalisierte Protestakteure, die sich selbst in expliziter Opposition gegenüber Staat und Regierung positionieren. Diese Organisationen spielen oft eine zentrale Rolle in den Konflikten, von denen riots ein Teil sind. Ich untersuche am Beispiel der food riots und Proteste gegen die hohen Lebenshaltungskosten in Burkina Faso 
ab 2008, wie Gewerkschaften und Bewegungsorganisationen sich auf riots beziehen. In den Narrativen der Protestakteure lassen sich zwei maßgebliche Argumente identifizieren. Die Gewerkschaften und ihre Verbündeten stellen die riots als Resultat ihrer eigenen jahrelangen Mobilisierungsarbeit dar, obgleich die Aufstände nicht von ihnen organisiert und an den riots andere soziale Gruppen beteiligt waren. Ein weiteres zentrales Element ist die Gegenüberstellung ihrer eigenen ,organisierten', ,kontrollierten' und ,friedlichen' Proteste, die ,klare Ziele' aufweisen würden, auf der einen Seite und der ,spontanen', ,unkontrollierten' und ,ziellosen' riots auf der anderen. Letztere werden mit Sachschäden und dem Fehlen von Organisationsstrukturen, Verantwortlichen und Führungspersonen in Verbindung gebracht. Dieser Dualismus spielt für die oppositionellen Organisationen eine wichtige Rolle: Er erfüllt die Funktion, sie selbst als legitime Verhandlungspartnerinnen gegenüber den staatlichen Akteuren und der Regierung zu positionieren.

Die Materialgrundlage der Fallstudie bilden Dokumente von internationalen und Nichtregierungsorganisationen, Presseberichte, Flugblätter, Zeitschriften und andere Materialien der Gewerkschaften und Bewegungsorganisationen sowie 35 leitfadengestützte Interviews, die zwischen Oktober und Dezember 2011 sowie im August und September 2012 an vier Standorten in Burkina Faso geführt wurden. Interviewt wurden Aktivist_innen und Funktionär_innen der Gewerkschaften und anderer Organisationen, an den riots Beteiligte sowie Vertreter_innen staatlicher Institutionen. Gegenstand der Interviews waren die riots und die Proteste eines Bündnisses ,gegen das teure Leben“, insbesondere die Entstehung der riots, die Mobilisierung zu den Protesten, die Forderungen und die spezifischen Formen des Protests.

Der Artikel ist wie folgt gegliedert: Der folgende Abschnitt skizziert zunächst kurz die sozialwissenschaftliche Debatte um riots. Ich beziehe mich hier insbesondere auf die marxistisch-strukturalistische Perspektive, die sich auch in den Narrativen der burkinischen Protestakteure widerspiegelt, sowie auf die Kritik an dieser Perspektive, wie sie mit Blick auf Proteste marginalisierter Akteur_innen formuliert wurde. Anschließend stelle ich die riots und Proteste gegen die hohen Lebenshaltungskosten in Burkina Faso dar. Ich analysiere, wie das dortige Bündnis aus Gewerkschaften und Bewegungsorganisationen das Thema der hohen Preise aufgriff und ihren Forderungen entsprechend rahmte. Dabei nutzten sie die riots als Gelegenheit zur Mobilisierung sowie als strategisches Element in ihrer Argumentation, um ihre eigene Position im Konflikt mit der burkinischen Regierung zu stärken.[1]

\section{Debatten um riots}

Eine Sicht in den politischen und sozialwissenschaftlichen Debatten um riots versteht diese als unpolitische, kriminelle Handlungen, insbesondere wenn sie mit Plünderungen und physischer Gewalt einhergehen. Die riots in London 2011 beispielsweise wurden in Teilen der öffentlichen Debatte als kriminell und explizit unpolitisch gerahmt (Lamble 2013). Auch die riots in den französischen Banlieues der 1990er Jahre sowie 2005 wurden als ,städtische Gewalt‘ und Teil eines „Dualismus von Stadt versus Banlieue“" (Dikeç 2004: 205) konstruiert; über diese Konstruktion wurden repressive Politiken und Praktiken von Verwaltung und Sicherheitskräften 
(beispielsweise die Ausweitung von Polizeibefugnissen, verdachtsunabhängige Kontrollen etc.) legitimiert. Dem steht eine andere Perspektive auf riots gegenüber, welche sie als explizit politische Phänomene beschreibt. Sie hebt die politischen Bedingungen und Kontexte hervor, aus denen heraus riots entstehen: wirtschaftliche, gesellschaftliche und politische Strukturen, die bestimmte Gruppen systematisch ausschließen und marginalisieren. Riots werden als Reaktion auf soziale und wirtschaftliche Missstände verstanden, gelten dabei aber als unorganisiert und ohne identifizierbare Ziele, folglich also nicht als kollektives politisches Handeln (vgl. Brown 2000; Wilkinson 2009). Diese Argumentationslinie entspricht einer marxistischen Perspektive, welche riots als entstanden aus sowie Reaktion auf ungleiche ökonomische und politische Verhältnisse begreift, dabei jedoch betont, dass die berechtigte Wut und Frustration der an riots Beteiligten von formellen Organisationen kanalisiert und organisiert werden müsse (Harvie/ Milburn 2013: 561). In der akademischen Debatte um riots wurde diese Position dafür kritisiert, dass sie Politik als linearen Prozess verstünde (etwa Balibar 2007). In Teilen der europäischen Linken hat der klassische marxistische Dualismus von Revolution und riot - wobei Erstere als intentional und geplant, Letztere als spontan und ,unorganisiert angenommen werden - an Bedeutung verloren. Die dominante Stellung des weißen männlichen Proletariats als vermeintlich wichtigstes und womöglich einziges Subjekt sozialer Kämpfe wurde politisch unter anderem durch Studierenden-, Frauen-, queere und migrantische Bewegungen infrage gestellt, theoretisch durch feministische und poststrukturalistische Perspektiven.

Die Perspektive auf riots als inhärent politische Phänomene bezieht sich teilweise auf diese theoretischen Zugänge. Riots werden als „Objekte von Politik“" (Trott 2013: 539) analysiert. Im Mittelpunkt dieser sich insbesondere auf Michel Foucault stützenden Analysen stehen die Machttechnologien sowie die Disziplinierungs- und Bestrafungspraktiken, denen rioter ausgesetzt werden. Zudem stehen die rioter selbst als aktiv Handelnde im Mittelpunkt. Gefragt wird beispielsweise danach, welches $\mathrm{Maß}$ an „Rationalität, Bewusstsein, geteilten Werten oder Moral erforderlich ist, um als politisches Subjekt zu gelten" (ebd.).

Es handelt sich dabei keineswegs um neue Fragen: $\mathrm{Zu}$ den ersten und nach wie vor einflussreichsten Autoren, die sich damit befasst haben, zählt etwa E. P. Thompson. In seiner Moral Economy of the English Crowd(1971) argumentierte Thompson gegen eine Sicht auf riots als vermeintlich unpolitisches Phänomen. Er zeigte, dass sich die food riots im Großbritannien des 18. Jahrhunderts, anders als im Mainstream der Geschichtsschreibung dargestellt, auf konkrete Vorstellungen von Legitimität und Gerechtigkeit gründeten - der Export von Getreide in Zeiten von Hunger und Nahrungsmittelknappheit wurde als zutiefst ungerecht und moralisch unvertretbar gesehen -, die der Entwicklung kapitalistischer Märkte zu dieser Zeit entgegen standen. Thompson kritisierte, dass die food riots in behavioristischer Weise als von Emotionen und Instinkten getrieben dargestellt wurden. Eine solche Darstellung ginge damit einher, den riotern ihren Status als aktiv handelnde politische Subjekte abzusprechen (Thompson 1971: 77).

In der sozialwissenschaftlichen Debatte fällt auf, dass riots in Feldern wie der Stadtforschung (etwa Harvey 2012; Wacquant 2008) mehr 
Aufmerksamkeit erfahren als in der Forschung zu sozialen Bewegungen und contentious politics[2]. Arbeiten aus dem Bereich der kritischen Stadtforschung analysieren riots beispielsweise aus der Perspektive von urban citizenship als Forderung nach gesellschaftlicher Teilhabe und politischdemokratischer Anerkennung (Amin 2003; Duprez 2009; Moran 2011). David Garbin und Gareth Millington (2012) zeigen am Beispiel der Pariser Banlieu La Courneuve, anschließend an Pierre Bourdieus Konzeption des sozialen Raums, wie sozialräumliche Marginalisierung in einer territorialen Stigmatisierung bestimmter städtischer Gruppen durchgesetzt und verfestigt wird und damit die strukturelle Hintergrundfolie für riots schafft. Andere Autor_innen binden, meist bezugnehmend auf Henri Lefebvre, riots als spezifisch städtische Protestform an die Debatte um das „Recht auf Stadt“ zurück (Harvey 2012; Mayer 2009; Mitchel 2003).

Häufig werden riots als durch zwei charakteristische Merkmale gekennzeichnet beschrieben: Spontaneität und physische Gewalt (etwa Arezki/ Brückner 2011; Lupsha 1969). Spontaneität impliziert das Fehlen strategischer Planung und Organisation sowie die offene Ansprache der Adressat_innen von Protest, potenzieller Anhänger_innen, Unterstützer_innen und Verbündeter. Infolge dessen werden physische Gewalthandlungen, die mit riots in Verbindung stehen, auch von der Bewegungs- und Protestforschung - anders als in anderen Fällen von physischer Gewalt im Zuge von politischem Protest - selten als taktische Repertoires gefasst, sondern als Ausdruck von Emotionen und fehlender rationaler strategischer Planung (vgl. Gamson 1975). Analysen aus dem Bereich der Bewegungs- und Protestforschung konzentrieren sich überwiegend auf kollektives Handeln von formalisierten Organisationen und identifizierbaren Gruppen (Snow et al. 2004). Aufbauend auf Theorien kollektiven Handelns stehen die Entstehungsund Erfolgsbedingungen für Mobilisierung und politischen Protest im Mittelpunkt dieser Forschungsrichtung. Die meisten Ansätze gehen dabei von einem Mindestmaß an organisatorischer Struktur als notwendiger Bedingung für Protest aus. Die prominenteste Kritik an dieser konzeptuellen Verknüpfung von Bewegung und Organisation haben Frances Fox Piven und Richard Cloward formuliert:

„Die Betonung der bewußten Intention [...] spiegelt die Verwechslung von Massenbewegungen mit formalisierten Organisationen, die in der Regel auf dem Höhepunkt der Bewegung auftauchen, wider die Verwechslung zweier zwar ineinander verwobener, aber dennoch ganz verschiedener Phänomene. [...] Was auch immer die intellektuellen Ursachen für diesen Irrtum sein mögen, die Gleichsetzung von Bewegung mit ihren Organisationen - die zudem voraussetzt, daß Proteste einen Führer, eine Satzung, ein legislatives Programm oder doch zumindest ein Banner haben müssen, bevor sie anerkannt werden - hat den Effekt, daß die Aufmerksamkeit von vielen Formen politischer Unruhe abgelenkt wird und diese per definitionem den verschwommenen Bereichen sozialer Probleme und abweichenden Verhaltens zugeordnet werden." (Piven/Cloward 1986: 29-30)

Auch James Scott (1987) warnt davor, Widerstand konzeptuell zu eng an Intentionalität und kollektives Handeln zu binden. Der Fokus auf offene und 
kollektive Formen des Protesthandelns, so Scotts Argument, liefe Gefahr, indirekt und ungewollt die Analyse auf die von den jeweiligen herrschenden Eliten definierten Räume und Arenen zu beschränken (ebd.).

In der folgenden Fallstudie zu den food riots und Protesten gegen die hohen Lebenshaltungskosten in Burkina Faso argumentiere ich, dass die kritischen Hinweise von Piven und Cloward sowie Scott nicht nur für externe Beobachter_innen relevant sind, sondern auch für die an den jeweiligen politischen Debatten um riots aktiv Beteiligten - gerade für diejenigen, die mit den riotern sympathisieren. Denn sie verfügen über Gestaltungsmacht, die Räume von Protest und kollektivem Handeln abzustecken und zu definieren, welchen Individuen und sozialen Gruppen welche Möglichkeiten zugestanden werden, ihre Interessen zu artikulieren.

\section{Food riots und Proteste „gegen das teure Leben in Burkina Faso"}

Der außerordentliche Anstieg der Nahrungsmittelpreise zählt zu den schwerwiegendsten Folgen der gegenwärtigen globalen Krisen. In der ersten Jahreshälfte 2008 erreichte der wichtigste Index für Nahrungsmittelpreise, der Food Price Index der FAO, Höchstwerte. Im März lag er mehr als 50 Prozent über dem Wert des Vorjahres. 2009 sanken die Preise zwar vorübergehend, stiegen 2011 jedoch wieder stark und haben sich seither auf hohem Niveau stabilisiert (FAO Homepage, 16.01.2016). In Dutzenden Städten weltweit - etwa in Kairo, Rabat, Mogadischu, Abidjan, Dakar, Nairobi, Port-au-Prince, Lima und Dhaka - protestierten die Menschen mit Hungeraufständen, Demonstrationen und Streiks gegen den Preisanstieg. Bei den Hungeraufständen handelte es sich meist um mehr oder weniger spontane, nicht angemeldete Demonstrationen. Vielerorts mobilisierten Gewerkschaften, Verbraucherverbände und Frauengruppen Tausende Menschen zu Demonstrationen, im Zuge derer es teilweise zu Besetzungen von Regierungsgebäuden oder Plünderungen von Geschäften und Nahrungsmittellagern kam. In vielen Fällen reagierten die Sicherheitskräfte mit Gewalt, zahlreiche Menschen wurden getötet oder verletzt. Der rasante Anstieg der Preise war eher ein Auslöser als die eigentliche Ursache der Auseinandersetzungen, bei denen es im Kern um sozioökonomische Ungleichheit, globale kapitalistische Verhältnisse und politische Unterdrückung ging. Häufig richteten sich Demonstrationen explizit gegen die jeweils herrschenden Parteien und Präsidenten, die in vielen Fällen schon seit Jahren oder Jahrzehnten an der Macht sind (Bush 2010; Harsch 2008; Patel/McMichael 2009).

Die Durchsetzung kapitalistischer Vergesellschaftung stellt eine Rahmenbedingung dar, ohne welche die Hungeraufstände nicht zu verstehen sind. Denn bei den Protesten geht es nicht um die Knappheit von Nahrung als solche: nicht darum, dass zu wenig Nahrungsmittel verfügbar wären, sondern darum, dass arme städtische Bevölkerungsschichten sie sich nicht leisten können. So ist auch die jüngste Nahrungsmittelpreiskrise nicht als singuläres Ereignis zu verstehen, das im Sinne eines externen Schocks die riots Anfang 2008 ausgelöst hätte. Sie ist vielmehr Ausdruck und Bestandteil eines historisch spezifischen Geflechts politisch-ökonomischer Beziehungen insbesondere zwischen dem globalen Norden und 
Süden. Das gegenwärtige globale Ernährungsregime zeichnet sich durch die Abhängigkeit des Industriekapitalismus von fossilen Energien ebenso aus wie durch die Expansion der Agrarkraftstoffproduktion, Spekulationen auf Boden und auf den Nahrungsmittelmärkten sowie die Konzentration und Zentralisierung der profitorientierten Produktion von Nahrungsmitteln (McMichael 2009). Wachsender Energiebedarf bei gleichzeitiger Erkenntnis, dass die weltweiten fossilen Energiereserven endlich sind, sowie der Wunsch nach Unabhängigkeit von den Ölförderstaaten haben dazu geführt, dass vor allem Industrie- und Schwellenländer zunehmend versuchen, ihren Energiebedarf durch Agrarkraftstoffe zu decken. Infolgedessen werden groBe Flächen im globalen Süden für den Anbau von entsprechenden Pflanzen (etwa Zuckerrohr, Jatropha oder Ölpalmen) umgenutzt - und stehen deshalb für die Produktion von Nahrungsmitteln nicht (mehr) zur Verfügung.

In internationalen medialen, politischen und humanitären Debatten wurden food riots überwiegend als spontane Aktionen verhandelt, als unmittelbare und verzweifelte Reaktionen auf den Preisanstieg. „Die steigenden Nahrungsmittelpreise verursachten eine Epidemie des Hungers, die zu den riots geführt hat [...]. Ohne Nahrung revoltieren die Menschen, sie emigrieren, oder sie sterben“,[3] erklärte die geschäftsführende Direktorin des Welternährungsprogramms, Josette Sheeran (WFP 2009). „Wird uns das Jahr 2013 soziale Unruhen in Brasilien, Streiks in China oder eine Revolution in Pakistan bringen?", fragte David Frum 2012 in einem CNNBeitrag und ergänzte: „Die Antwort lässt sich wahrscheinlich aus den Preisindizes ableiten" (Frum 2012). Jacques Diouf, Generaldirektor der Food and Agriculture Organisation (FAO), warnte in seiner Rede auf der UNKonferenz für Ernährungssicherung, Klimawandel und Bioenergie im Juni 2008, die Welternährungskrise würde zu riots führen, „die den Frieden und die Sicherheit auf der Welt gefährden können“ (Diouf 2008: 1). Eine tiefer gehende Analyse dieser Diskurse ist nicht der Anspruch dieses Beitrags. Doch die angeführten Beispiele werfen zumindest Schlaglichter darauf, wie food riots im Zuge der jüngsten Nahrungsmittelpreiskrise als tragischer Ausdruck einer humanitären Krise dargestellt wurden.

In Burkina Faso fanden Ende Februar 2008 innerhalb einer Woche in den meisten größeren Städten des Landes Hungeraufstände[4] statt. Bei unangemeldeten (Bobo-Dioulasso, Ouahigouya) beziehungsweise nicht genehmigten (Ouagadougou) Demonstrationen kam es zu umfangreichen Sachschäden an Tankstellen, an öffentlichen und privaten Gebäuden. Bei Zusammenstößen zwischen Protestierenden und Sicherheitskräften wurden zahlreiche Menschen verletzt und Hunderte festgenommen (Harsch 2009; Maccatory et al. 2010). Die Protagonist_innen waren größtenteils marginalisierte urbane Jugendliche[5]; mehrheitlich junge Männer, die ohne regelmäBiges Einkommen im informellen Sektor ihr Überleben zu sichern versuchten.

Ende Februar 2008, am Tag der Hungeraufstände in Ouagadougou, versammelten sich die Gewerkschaftsführungen und verabschiedeten eine Erklärung, in der sie die entstandenen Schäden bedauerten, jedoch betonten, dass die Aufstände Ausdruck der legitimen Wut der Bevölkerung angesichts der gestiegenen Lebenshaltungskosten seien. Sie kritisierten die von der Regierung angekündigten Maßnahmen als ungenügend und riefen andere zivilgesellschaftliche Organisationen zu einem Treffen am 6. März 2008 und 
zu einer zentralen Kundgebung in Ouagadougou in der Folgewoche auf. Am 12. März schlossen sich die großen Gewerkschaften mit Konsumenten- und Berufsverbänden, Menschenrechtsorganisationen sowie Vereinigungen von Schüler_innen und Studierenden zur „Nationalen Koalition gegen das teure Leben, die Korruption, den Betrug, die Straflosigkeit und für die Freiheiten“ (Coalition nationale de lutte contre la vie chère, la corruption, la fraude, l'impunité et pour les libertés, CCVC) zusammen (CCVC 2008a). Das Bündnis organisierte zwei landesweite Generalstreiks im April 2008 und mehrere Großkundgebungen. Angeführt von den Gewerkschaften war die CCVC die treibende Kraft in der Mobilisierung zu den Protesten gegen die hohen Lebenshaltungskosten in Burkina Faso. Für die Gewerkschaften lag es nahe, das Thema der gestiegenen Preise unmittelbar im Anschluss an die Hungeraufstände aufzugreifen und zur Mobilisierung zu nutzen, weil dieses schon seit einigen Jahren auf ihrer Agenda stand. Bereits eine Woche vor den Aufständen hatte der gewerkschaftliche Dachverband Confédération générale des travailleurs du Burkina (CGT-B) eine Erklärung veröffentlicht, in der er die ,anderen Organisationen (der Studierenden, der Konsument_innen und der Menschenrechtsbewegung, der Frauen, der Jugend...)“(CGT-B 2008: 1) zu gemeinsamen Initiativen aufrief.

Ab März 2008 waren die außerparlamentarischen oppositionellen Akteure federführend in den Protesten gegen die hohen Lebenshaltungskosten, insbesondere die Gewerkschaften, Menschenrechts- und Studierendenorganisationen. In diesen Organisationen sind vor allem Mittelschichtsangehörige in den Städten vertreten: Studierende, Schüler_innen weiterführender Schulen und abhängig Beschäftigte. In den Forderungen der CCVC spiegelt sich auch die zentrale Rolle der Gewerkschaften und ihrer Hauptklientel, die aus Angestellten des öffentlichen Dienstes im Bildungsund Gesundheitsbereich besteht, wider. So steht im Forderungskatalog der Koalition an erster Stelle „eine Erhöhung der Gehälter und Pensionen der Staatsbediensteten sowie der Arbeiter_innen im privaten Sektor“ (CCVC 2008b). Und als einen zentralen Erfolg ihrer Proteste erklären Vertreter_innen des Bündnisses die Herabsetzung der Lohnsteuer und die Erhöhung der Gehälter im öffentlichen Dienst.[6]

Hingegen waren an den food riots vom Februar 2008 zahlreiche Angehörige sozialer Gruppen beteiligt, die in den Gewerkschaften und den etablierten Bewegungsorganisationen nur schwach vertreten sind. Die Mobilisierung zu den riots verlief in den informellen Netzwerken dieser Jugendlichen, über persönlichen Kontakt und SMS. Formale Organisationsstrukturen bestehen kaum. Riots als typisches Protestrepertoire dieser Gruppen (vgl. Alexander/Pfaffe 2013; Engels 2015) haben in den burkinischen Städten eine Tradition aus früheren Konflikten (Harsch 2009). Im Jahr 2006 kam es beispielsweise in Ouagadougou zu riots, als die Regierung versuchte, eine Helmpflicht für Mopeds und Mofas durchzusetzen (Chouli 2012: 134). Nichtsdestotrotz zeigten sich die meisten Beobachter_innen, auch innerhalb der Gewerkschaften und Bewegungsorganisationen, von den food riots im Februar 2008 überrascht.[7]

Die burkinische Regierung und die Behörden reagierten unmittelbar auf die Hungeraufstände zunächst mit dem Einsatz der Sicherheitskräfte und zahlreichen Verhaftungen. Darüber hinaus ergriffen sie eine Reihe von 
Maßnahmen, um die Auswirkungen der Preiskrise abzufedern. Sie setzte zeitweise die Importzölle und Mehrwertsteuer auf Grundnahrungsmittel aus und errichtete Ausgabestellen für subventionierte Lebensmittel (Africa Research Bulletin 2008; Assemblée Nationale 2008; Chouli 2012; Zahonogo et al. 2011). Die ersten Maßnahmen wurden kurz nach den Hungeraufständen im März 2008 angekündigt, andere erfolgten später nach erneuten Protesten.

Obwohl die Nahrungsmittelpreiskrise und die riots vom Februar 2008 die Auslöser für die Gründung waren, war die CCVC nur teilweise ein neues Bündnis. Die Allianz baute institutionell und personell stark auf eine bereits bestehende Koalition auf: das „Kollektiv der demokratischen Massenorganisationen und politischen Parteien“ (Collectif d'organisations démocratiques de masse et de partis politiques). Das Kollektiv war Anfang 1999 von Gewerkschaften, Menschenrechts- und Studierendenverbänden, Oppositionsparteien, Frauen- und Anwaltsorganisationen gegründet worden. Anlass war die Ermordung des regimekritischen Journalisten Norbert Zongo im Dezember 1998 (Frère 2010; Harsch 2009; Hilgers 2010). Beide Bündnisse, das Kollektiv und die CCVC, bestehen nach wie vor parallel und mit großen personellen und institutionellen Überschneidungen. Sie unterscheiden sich insofern, als bei der CCVC die marxistisch orientierte CGT-B, der mitgliederstärkste Gewerkschaftsverband des Landes, die Federführung hat, während das Kollektiv vom Mouvement burkinabè des droits de l'homme et des peuples (MBDHP), der einflussreichsten Menschenrechtsorganisation, angeführt wird. Der Vorsitzende des MBDHP ist zugleich Sprecher des Kollektivs und Vizepräsident der CCVC. Ein weiterer Unterschied besteht darin, dass in der CCVC politische Parteien ausgeschlossen sind. Vertreter des Bündnisses erklären dies mit dem Verweis darauf, dass man im Kollektiv die Erfahrung gemacht hätte, dass Parteipolitiker_innen die zivilgesellschaftlichen Allianzen für individuelle Machtinteressen missbrauchen könnten.[8]

\section{Diskursive Rahmung der riots}

Die Gewerkschaften und die mit ihnen verbündeten Organisationen schlossen in ihrer Mobilisierung unmittelbar an die food riots an. Sie rahmten den Preisanstieg ihren zentralen Forderungen (etwa nach der Erhöhung der Gehälter) entsprechend und stellten die riots teilweise als Erfolg ihrer eigenen, voran gegangenen Mobilisierung dar. In ihren Narrativen nutzten sie die riots als Referenzpunkt, um ihre eigenen Strategien und Forderungen zu legitimieren und sich selbst als Verhandlungspartnerinnen der nationalen Regierung zu positionieren. Vertreter_innen von CCVC-Mitgliedsorganisationen ebenso wie von staatlichen Institutionen argumentierten in den Interviews, zu den food riots vom Februar 2008 sei es nur gekommen, weil die im Kollektiv zusammengeschlossenen Organisationen zuvor jahrelange kontinuierliche Mobilisierungsarbeit betrieben hätten.

„Die Leute haben genug davon, und sie reagieren. Das ist das Ergebnis von Jahren der Arbeit zur politischen Bewusstseinsbildung durch das Kollektiv. Das Kollektiv hat großartige Arbeit geleistet. [...] Heute lassen die Leute sich nicht mehr alles einfach so gefallen. Die spontanen Bewegungen sind kein Vandalismus, sie sind politisches Bewusstsein!“ 
So äußerte sich ein CCVC-Vertreter[9]. Diese Interpretation spiegelt ein typisches marxistisches Verständnis von riots wider. Dies ist insofern wenig überraschend, als viele der in der CCVC zusammengeschlossenen Organisationen (auch jene, welcher der zitierte Vertreter angehört), marxistisch orientiert sind. Damit einher geht das Selbstverständnis einiger Organisationen, die es als ihre Aufgabe sehen, die ,Massen“ zu organisieren und für die Revolution zu mobilisieren: „Der Kampf wird von den Massen und für die Massen gemacht. [...] Wir verstehen uns als demokratische Massenorganisation; unser Platz ist an der Seite des Volkes“, erläuterte ein Aktivist der Studierendenbewegung[10]. Ein anderer erklärte, dass „die Massen wegen der hohen Preise auf die Straßen gegangen sind“, jedoch sei diese „spontane Bewegung“ nicht gut organisiert gewesen, weswegen die Gründung der CCVC erfolgt sei: „Die Organisationen reagieren auf die Wünsche und Probleme der Massen.“[11].

In der Darstellung durch die CCVC und ihre Mitgliedsorganisationen lässt sich eine Gegenüberstellung der riots und ihrer eigenen Protestaktionen ausmachen: Während hier die Ersteren unkontrolliert und potenziell gewaltsam erscheinen, werden die Zweiteren als friedlich, kontrolliert, gut organisiert und konform mit den Regularien des Versammlungsrechts gezeigt. In der Konstruktion sowohl der Gewerkschaften und Bewegungsorganisationen als auch ihrer staatlichen Gegenspieler (Regierung, Verwaltungen, Sicherheitskräfte) besteht ein Dualismus von Marsch (marche[12]) gegenüber Aufstand (émeute). Marsch meint eine geplante, gut organisierte, dem Versammlungsrecht entsprechend angekündigte Demonstration, die von identifizierbaren kollektiven Akteuren organisiert wird sowie in geregelten Bahnen und ohne Probleme verläuft. Dabei sind mit Problemen vor allem Sachschäden und gewaltsame Auseinandersetzungen zwischen Sicherheitskräften und Demonstrant_innen gemeint. Demgegenüber werden riots als spontane und unorganisierte Aktionen dargestellt, die potenziell mit Sachschäden (casses[13]) verbunden sind (unabhängig davon, ob diese intendiert sind oder nicht). „Ein riot ist es, wenn die Leute einfach rausgehen, um etwas kaputt zu machen“, erklärte ein Aktivist, „eine Demonstration ist es, wenn es klare Ziele gibt."[14]

Es besteht eine grundlegende Übereinstimmung zwischen den oppositionellen Organisationen und den staatlichen Akteuren über den Gegensatz von ,friedlichem Protest' und ,casses', auf den sich beide in der Verhandlung ihrer politischen Auseinandersetzung beziehen. In den Diskursen und Praktiken von contentious politics werden formelle und informelle Regeln darüber, wie politische Konflikte ausgetragen und Forderungen artikuliert werden, zwischen herrschenden und oppositionellen Akteuren ausgehandelt, produziert, angefochten und reproduziert. Im burkinischen Beispiel akzeptieren staatliche und oppositionelle Akteure die „Tatsache“, dass es zu gewaltsamen Konfrontationen kommt, wenn staatliche Sicherheitskräfte bei Protestaktionen einschreiten. Bei den „kontrollierten“ Demonstrationen der Gewerkschaften und anderen Organisationen gegen die hohen Lebenshaltungskosten hielten sich die Sicherheitskräfte deshalb weitgehend im Hintergrund; bei den „spontanen Bewegungen“ „müssten“ die Behörden die Sicherheitskräfte schicken, argumentieren sowohl Behörden- als auch Bewegungsvertreter_innen[15]. Ein Gewerkschafter berichtet, bei der 
Großdemonstration der CCVC am 8. April 2011 hätte er selbst die Gendarmerie gerufen, um ihr eine Person zu übergeben, die versucht hätte, Sachschäden zu herbeizuführen[16]. Ein anderer erklärte:

„Die Gewerkschaftsführungen wissen, wie man Protestaktionen kontrolliert, so dass es nicht zu gewaltsamen Ausschreitungen kommt. Unser Vorteil als formale Struktur ist, dass wir casses und andere Störungen der öffentlichen Ordnung vermeiden können. Wenn es casses gibt, müssen die Sicherheitskräfte den öffentlichen Frieden wieder herstellen - mit all den Problemen, die damit einher gehen, dass Leute verletzt oder getötet werden.“[17]

Johanna Siméant (2011) stellt in ihrer Analyse von Auseinandersetzungen in der malischen Hauptstadt Bamako eine Delegitimierung von Protest durch den Rekurs auf Gewalt fest. Sie weist darauf hin, dass eine vereinfachende Gegenüberstellung von, gewaltsamen' und, friedlichen' Protesten analytisch wenig hilfreich ist, hingegen der Blick auf das Verhältnis unterschiedlicher Protestformen Aufschluss über den Verlauf sozialer Auseinandersetzungen geben kann. Denn Aufstände und formalisierter Protest sind häufig diskursiv miteinander verknüpft; so beziehen sich Narrative über ,reguläre Proteste implizit oder explizit auf die Möglichkeit ,unorganisierter Auseinandersetzungen (Siméant 2011: 2). Im Fall der burkinischen Proteste „gegen das teure Leben“ verweisen die Gewerkschaften und zivilgesellschaftlichen Organisationen einhellig darauf, dass es zu den Aufständen, die mit einigen Sachschäden einhergingen, nicht gekommen wäre, wenn die Regierung sich nach früheren gewerkschaftlichen Protesten auf größere Zugeständnisse hinsichtlich von Preiskontrollen und einer Erhöhung der Kaufkraft eingelassen hätte. Insofern trifft Siméants auf Mali bezogene Beobachtung für Burkina Faso nicht zu. Denn dort wurden durch den Verweis auf die Aufstände die Aktionen der Gewerkschaften und anderen Organisationen argumentativ aufgewertet - und zwar ebenso durch diese Organisationen selbst wie durch ihre staatlichen Gegenspieler. Die CCVC bezieht sich strategisch auf die riots, um sich selbst als relevante Verhandlungspartnerin der Regierung zu positionieren. Bis 2008 sei die Regierung gegen sie gewesen, erklärte einer der führenden Aktivisten des Bündnisses. Nach den „,spontanen Aufständen“ hätten die Vertreter des Staates verstanden, ,wenn es das Kollektiv oder die CCVC ist, ist alles unter Kontrolle, und man weiß, wer die Verantwortlichen sind. Wenn wir demonstrieren, haben wir klare Forderungen."[18]

Im Anschluss an Herbert Haines (1984) lässt sich diese Strategie als „Flankeneffekt“ beschreiben. Haines analysiert, welche Folgen es für eine soziale Bewegung hat, wenn sich Teile von ihr radikalisieren, und benennt sowohl positive wie negative „radical flank effects“. Als negativen Flankeneffekt bezeichnet er, wenn die externe Unterstützung auch für die moderaten Flügel einer Bewegung infolge radikaler Aktionen schwindet. Demgegenüber ist ein positiver Flankeneffekt zu beobachten, wenn die Existenz radikaler Gruppen und ihre Aktivitäten die Verhandlungsposition der Moderaten stärken. Ein solcher Effekt kann entweder eintreten, weil radikale Aktionen eine Krise verursachen, die zum Vorteil der Moderaten gelöst wird; oder weil radikale Gruppen eine Kontrastfolie bieten, gegenüber der die Forderungen und Strategien moderater Akteure diskursiv normalisiert und als „vernünftig“ 
dargestellt werden können (Haines 1984: 32). Letzteres ist bei den burkinischen Hungeraufständen und Protesten „gegen das teure Leben“ der Fall. Die Hungeraufstände 2008 haben die Position der Gewerkschaften und anderer etablierter Organisationen im Machtkampf mit der Regierung verbessert und sie eher legitimiert als delegitimiert. Die Gewerkschaften seien seither in einer stärkeren Position, weil sie sich im „legalen Rahmen“ bewegen würden, so ein Gewerkschaftsvorsitzender: „Die informellen Gruppen respektieren das Gesetz nicht. [...] Die Regierung hat [jetzt] verstanden, dass sie die Forderungen der Gewerkschaften lieber anhören sollte[19].“ Der positive Flankeneffekt wird im burkinischen Beispiel - im Unterschied zu Haines’ Untersuchung - nicht durch eine Radikalisierung innerhalb einer Bewegung hervorgerufen, sondern durch riots - Aufstände von Angehörigen sozialer Gruppen, die in den entsprechenden Bewegungen und Organisationen wenig vertreten sind.

Die soziale Basis der Proteste gegen die hohen Lebenshaltungskosten war breiter als die früherer Auseinandersetzungen. So schlossen sich beispielsweise zahlreiche kleine Händler_innen und ihre Vereinigungen auf den lokalen Märkten den Aktionen der CCVC an. Trotzdem ist es dem Bündnis kaum gelungen, die große Gruppe der erwerbslosen Jugendlichen und im informellen Sektor Tätigen zu integrieren, die in den riots eine zentrale Rolle einnahmen. Das Problem der Repräsentation und Organisierung solcher gesellschaftlich marginalisierter Gruppen ist linken Bewegungen und Gewerkschaften in Europa und Nordamerika vertraut, deren Klientel ebenfalls in den abhängig Beschäftigten, Studierenden und städtischen Mittelschichten besteht und die über wenig Verankerung im subproletarischen Milieu, unter Wohnungslosen oder Illegalisierten verfügen. Auch die Gewerkschaften und Bewegungen in Burkina Faso sind sich dieses Problems sehr bewusst: „Auch deswegen haben wir die CCVC gegründet“, erklärte ein Verantwortlicher des Bündnisses.[20]

\section{Schlussbemerkung}

Als es im Zuge der globalen Nahrungsmittelpreiskrise in Burkina Faso Anfang 2008 zu Hungeraufständen kam, nahmen die Gewerkschaften und Bewegungsorganisationen das Thema unmittelbar im Anschluss an die riots auf und mobilisierten zahlreiche Menschen zu Generalstreiks, Großdemonstrationen und anderen Protestaktionen. Der Anstieg der Lebensmittelpreise bietet ein besonderes Potenzial für umfassende Mobilisierung, weil er große Teile der Bevölkerung in ähnlicher Weise betrifft. Für die ärmsten Schichten hat der Preisanstieg eine existenzbedrohende Wirkung, für die Mittelschichten verstärkt er die relative Deprivation. Die Proteste gegen die hohen Preise waren deshalb nicht wie andere Mobilisierungen auf ein relativ kleines Bevölkerungssegment beschränkt, wenngleich an unterschiedlichen Protestformen (Hungerrevolten, Demonstrationen der CCVC) unterschiedliche Gruppen beteiligt waren.

Die Proteste „gegen das teure Leben“ sind ein Beispiel intensiver und erfolgreicher Mobilisierung durch ein breites Bündnis oppositioneller zivilgesellschaftlicher Gruppen. Sie sind aber auch ein Beispiel für die Ambivalenzen von Protest, insbesondere hinsichtlich der Fragen von Repräsentation und Organisierung sowie des Verhältnisses von ,organisiertem‘ und ,unorganisiertem'kollektivem Handeln. Riots entziehen sich etablierten Mustern des 
Protests; in vielen Fällen verweigern rioter entsprechende Interpretationsund Kommunikationsangebote - auch seitens linker Sympathisant_innen. Ein positiv konnotiertes Verständnis von riots als spontane Reaktion marginalisierter Akteur_innen auf tief greifende soziale und ökonomische Ungleichheit und Ungerechtigkeit ist unter linken Aktivist_innen und Organisationen weit verbreitet, in Afrika und Europa gleichermaßen.

Im Fall der Proteste gegen die hohen Lebenshaltungskosten in Burkina Faso stellten die riots eine Gelegenheit dar, welche die etablierten Protestakteure erfolgreich zur Mobilisierung nutzen konnten. Indem sie auf ihre bestehenden Netzwerke und Erfahrungen aufbauten und das Thema anschlussfähig an ihre vorhandenen Forderungen rahmten, gelang es ihnen, ihre Klientel zu mobilisieren und die soziale Basis ihrer Proteste weiter zu vergrößern. Auf diese Weise konnten die Protestorganisationen in Burkina Faso über einen längeren Zeitraum hinweg ein hohes Maß an Mobilisierung aufrecht erhalten und die Regierung unter Druck setzen. Eine erfolgreiche Strategie war dabei die Konstruktion des Dualismus von riots als unorganisiert, unkontrolliert und potenziell gewaltsam gegenüber den organisierten, kontrollierten und friedlichen Protesten der CCVC. Diese Konstruktion ist kohärent mit der marxistischen Ideologie, an der sich viele der in der CCVC zusammengeschlossenen Organisationen orientieren. Eine marxistischstrukturalistische Sicht auf riots und ein Verständnis von Protestierenden als aktiv Handelnden schließen sich keineswegs aus. Beispielsweise beschreibt Ray Bush food riots als

„Teil des Widerstands gegen Globalisierung und die ungleiche Verteilung kapitalistischer Produktionsverhältnisse. Sie sind pragmatische und üblicherweise unorganisierte, aber extrem effektive Ausdrucksformen von städtischen und ländlichen gesellschaftlichen Klassen, die gegen die Art und Weise protestieren, in der ihre livelihoods transformiert werden." (Bush 2010: 121)

Eine solche Konzeption muss nicht notwendigerweise auf der Annahme beruhen, dass jegliches politisches Handeln kollektiv und intentional, organisiert und kontrolliert ist, identifizierbare Akteure und offen formulierte Ziele und Forderungen aufweist. Die food riots von 2008 bestätigen vielmehr, was Thompson für jene feststellt, die mehr als 200 Jahre zuvor stattfanden: dass sie nicht notwendigerweise eines hohen Grades an Organisierung bedürfen (Thompson 1971: 119). Nahrungs- und Nahrungsmittelpreiskrisen haben ihre Ursachen in politisch-ökonomischen Strukturen. Riots und der organisierte Protest oppositioneller Organisationen stellen zwei Seiten derselben Medaille dar, unterschiedliche Akteure mit unterschiedlichen Protestrepertoires, die sich gegen das gleiche strukturelle Problem wenden.

\section{Endnoten}

[1] Vielen Dank an die Redaktion von s u b / u r b a n und insbesondere die Schwerpunktredakteur_innen dieses Hefts sowie an die Gutachter_innen für zahlreiche, sehr hilfreiche Hinweise

[2] Contentious politics bezeichnet die Schnittmenge von contention, politics und collective action. Konzeptionell verbirgt sich hinter dem Terminus die Idee, dass widerständiges 
politisches Handeln vielfältige Formen annehmen kann - reformorientierte Prozesse ebenso wie soziale Bewegungen oder revolutionäre Bestrebungen (Tilly/Tarrow 2007).

[3] Alle Zitate aus dem Englischen und Französischen wurden von der Autorin übersetzt.

[4] Die Beteiligten sprechen selbst von den Ereignissen als „Hungeraufständen“ (,émeutes de la faim').

[5] ,Jugendlich“ weniger im Sinne des Lebensalters als vielmehr als soziale Kategorie.

[6] L'Observateur Paalga 28.04.2012; Interviews mit Vertreter_innen von CCVC-Mitgliedsorganisationen in Banfora am 24.11.2011 sowie in Ouagadougou am 03.12.2011 und 02.09.2012.

[7] Interviews mit Vertreter_innen von Gewerkschaften in Ouagadougou am 10.11.2011; Interviews mit Vertreter_innen von Gewerkschaften und Menschenrechts- und Studierendenorganisationen in Bobo-Dioulasso am 25.11.2011.

[8] Interviews in Ouagadougou am 16.11.2011 und Koudougou am 08.12.2011.

[9] Interview in Ouagadougou am 02.09.2012.

[10] Interview in Ouagadougou am 16.11.2011.

[11] Interview in Ouagadougou am 16.11.2011.

[12] Im westafrikanischen Sprachgebrauch ist der Begriff „marche“für eine reguläre Demonstration üblicher als die Bezeichnung „manifestation“(die ebenfalls gebräuchlich ist).

[13] Casse ist die Substantivierung des französischen Verbs casser, das wörtlich „brechen“ oder „zerbrechen“ bedeutet. In der burkinischen Debatte, auf die ich mich hier beziehe, wird es als Oberbegriff für alle Gewalt gegen Sachen und Personen seitens der Protestierenden gebraucht, insbesondere gezielte oder unbeabsichtigte Beschädigungen an Gebäuden, etwa durch Steinwürfe oder Feuer.

[14] Interview in Bobo-Dioulasso am 25.11.2011.

[15] Interviews mit Behördenangehörigen in Banfora am 22.11.2011 und mit Mitgliedern einer Studierendenbewegung in Banfora am 24.11.2011.

[16] Interview am 05.12.2011

[17] Interview mit Gewerkschaftsmitgliedern in Ouagadougou am 01.12.2011.

[18] Interview am 16.11.2011.

[19] Interview in Ouagadougou am 10.11.2011

[20] Interview in Ouagadougou am 02.09.2012

\section{Autor innen}

Bettina Engels ist Politikwissenschaftlerin. Sie forscht zu Konflikten um Land und Bergbau sowie zu contentiuos politics vor allem in Afrika südlich der Sahara. Ihr theoretischer Schwerpunkt liegt auf Raum- und Handlungstheorie.

bettina.engels@fu-berlin.de

\section{Literatur}

Africa Research Bulletin (2011): BURKINA FASO: Unprecedented protests. In: Africa Research Bulletin: Political, Social and Cultural Series 48/4, 18793A-18794C.

Alexander, Peter / Pfaffe, Peter (2013): Social relationships to the means and ends of protest in South Africa's ongoing rebellion of the poor: The Balfour Insurrections. In: Social Movement Studies 13/2, 204-221.

Amin, Ash (2003): Unruly strangers? The 2001 urban riots in Britain. In: International Journal of Urban and Regional Research 27/2, 460-463.

Arezki, Rabah / Brückner, Markus (2011): Food prices, conflict, and democratic change. In: The University of Adelaide School of Economics Research Paper No. 2011-04.

Assemblée National (2008): Rapport de la Commission ad hoc sur la vie Chère. Ouagadougou: L’Assemblée Nationale du Burkina Faso. 
Balibar, Etienne (2007): Uprisings in the banlieues. In: Constellations 14/1, 47-71.

Brown, Rupert (2000): Group Processes: Dynamics Within and Between Groups. Oxford: Blackwell.

Bush, Ray (2010): Food riots: poverty, power and protest. In: Journal of Agrarian Change 10/1, 119-129.

CCVC (2008a): Acte de naissance de la CCVC, 12 March 2008. Ouagadougou: CCVC.

CCVC (2008b): Platform revendicative, 15 July 2008. Ouagadougou: CCVC.

CGT-B (2008): CGT-B declaration, 14 February 2008, 6 October 2008. Ouagadougou: CCVC.

Chouli, Lila (2012): Peoples' revolts in Burkina Faso. In: Firoze Manji (Hg.), African Awakening: The Emerging Revolutions. Cape Town: Pambazuka, 131-146.

Dikeç, Mustafa (2004): Voices into noises: Ideological determination of unarticulated justice movements. In: Space and Polity 8/2, 191-208.

Diouf, Jacques (2008): Speech at the UN Conference on Food Security, Challenges of Climate Change and Bioenergy, June 2008. Rome: FAO/UN.

Duprez, Dominique (2009): Urban rioting as an indicator of crisis in the integration model for ethnic minority youth in France. In: Journal of Ethnic \& Migration Studies 35/ 5, 753-770.

Engels, Bettina (2015): Different means of protest, same causes: popular struggles in Burkina Faso. In: Review of African Political Economy 42/143, 92-106.

Frère, Marie-Soleil (2010): „Enterrement de première classe“ ou „leçon de droit“: La presse burkinabè et l'affaire Norbert Zongo. In: Mathieu Hilgers / Jacinthe Mazzocchetti (Hg.), Révoltes et oppositions dans un régime semi-autoritaire. Le cas du Burkina Faso. Paris: Karthala, 241-267.

Frum, David (2012): Why 2013 will be a year of crisis. CNN, 03.09.2012. http://edition.cnn. com/2012/o9/o3/opinion/frum-food-price-crisis (letzter Zugriff am 23. Juni 2015).

Gamson, William A. (1975): The Strategy of Social Protest. Homewood (Illinois): Dorsey.

Garbin, David / Millington, Gareth (2012): Territorial stigma and the politics of resistance in a Parisian banlieue: La courneuve and beyond. In: Urban Studies 49/10, 2067-2083.

Haines, Herbert H. (1984): Black radicalization and the funding on civil rights: 1957-1970. In: Social Problems 32, 31-41.

Harsch, Ernest (2008): Price protests expose state faults. Rioting and repression reflect problems of African governance. In: Africa Renewal 22/2, 15.

Harsch, Ernest (2009): Urban protest in Burkina Faso. In: African Affairs 108/431, 263-288.

Harvey, David (2012): Rebel Cities: From the Right to the City to the Urban Revolution. London: verso.

Harvie, David / Milburn, Keir (2013): The moral economy of the English crowd in the twenty-first century. In: The South Atlantic Quarterly 112/3, 559-567.

Hilgers, Mathieu (2010): Evolution of political regime and evolution of popular political representations in Burkina Faso. In: African Journal of Political Science and International Relations 4/9, 350-359.

Lamble, Sara (2013): The quiet dangers of civilized rage: Surveying the punitive aftermath of England's 2011 riots. In: The South Atlantic Quarterly 112/3, 577-585.

Lupsha, Peter A. (1969): On theories of urban violence. In: Urban Affairs Review 4, 273-296.

Maccatory, Bénédicte / Oumarou, Makama Bawa / Poncelet, Marc (2010): West African social movements ,against the high cost of living“: From the economic to the political, from the global to the national. In: Review of African Political Economy 37/125, 345-359.

Mayer, Margit (2009): The ,Right to the City' in the context of shifting mottos of urban social movements. In: City, 13/2-3, 362-374.

McMichael, Philip (2009): A food regime analysis of the 'world food crisis'. In: Agric Hum Values, 4, 281-295.

Mitchell, Don (2003): The Right to the City: Social Justice and the Fight for Public Space. New York: Guilford.

Moran, Matthew (2011): Opposing exclusion: The political significance of the riots in French suburbs (2005-2007). In: Modern \& Contemporary France 19/3, 297-312.

Patel, Raj / McMichael, Philip (2009): A political economy of the food riot. In: review xxxii/1, 9-35.

Piven, Frances Fox / Cloward, Richard A. (1986 [1977]): Aufstand der Armen. Frankfurt am Main: suhrkamp. 
Scott, James (1987): Resistance without protest and without organization: Peasant opposition to the islamic zakat and the christian tithe. In: Comparative Studies in Society and History 29/3, 417-452.

Siméant, Johanna (2011): „Oh no! Let’s march but not riot!“ Street protests in Bamako during the years 1992-2010. Paper presented at the 4th European Conference on African Studies (ECAS 4). Uppsala, 17 June 2011.

Snow, David A. / Soule, Sarah A. / Kriesi, Hanspeter (2004): Mapping the terrain. In: David A. Snow / Sarah A. Soule / Hanspeter Kriesi (Hg.): The Blackwell Companion to Social Movements. Malden (Massachusetts): Blackwell, 3-16.

Thompson, Edward Palmer (1971): The moral economy of the English crowd in the eighteenth century. In: Past and Present 50, 76-136.

Tilly, Charles/Tarrow, Sidney G. (2007): Contentious Politics. Boulder und London: Paradigm.

Trott, Ben (2013): Introduction. Rebellious subjects: The politics of England's 2011 riots. In: The South Atlantic Quarterly 112/1, 538-540.

Wacquant, Loïc (2008): Urban Outcasts: A Comparative Sociology of Marginality. Cambridge: Polity.

WFP 2009: WFP Commends G8 Leaders' Focus on Food Security; Urges Support for Hunger Needs of World's Poorest. Press statement, published on 07 July 2009. http://www.wfp. org/news/news-release/wfp-commends-g8-leaders-focus-food-security-urges-supporthunger-needs-worlds-poorest [letzter Zugriff am 23. Juni 2015].

Wilkinson, Steven I. (2009): Riots. In: Annual Review of Political Science 12, 329-343.

Zahonogo, Pam /Bitibale, Soumaila / Kabre, Adama (2011): Etude sur la structure des prix des biens et services de grande consummation. Ouagadougou: Ministère de l’industrie, du commerce et de l'artisanat.

\section{Food riots and protest against high prices in Burkina Faso 2008}

Related to the recent food prices crisis, many African cities witnessed food riots. The article argues that food riots are often presented as spontaneous and violent-a reaction to social and economic grievances but still unorganised and aimless. This framing has been produced not only by "Western" media but also by protest actors in the Global South. The article focuses on struggles against high prices in Burkina Faso. Labour unions and social movement organisations used the food riots as an occasion to mobilise in favour of their own agendas. Food riots and trade union protests differed with respect to their protest repertoires and the social groups involved. Equipped with material and access to institutions, trade unions and social movement organisations took up the "spontaneous movements" and strategically referred to them in order to enhance their own strategies and claims. 
